\title{
Broadband and Small-size 3-dB Ring Coupler
}

\author{
Stefan Simion $^{1,}{ }^{*}$ and Giancarlo Bartolucci ${ }^{2}$
}

\begin{abstract}
A topology for a 3-dB broadband and small-size ring coupler is proposed. It consists of fully-distributed Composite Right-/Left-Handed phase shifters and a Lange coupler. For the fabricated coupler, the frequency bandwidth is one octave, centered on $1.5 \mathrm{GHz}$, while the footprint area is reduced to $25 \%$, compared to the conventional ring coupler topology. The experimental results are in good agreement with the expected ones, obtained by electromagnetic simulation.
\end{abstract}

\section{INTRODUCTION}

Since the conventional topology of the ring coupler has been proposed [1], other fully-distributed ring coupler configurations have been developed in order to reduce the printed area [2-6], to increase the frequency bandwidth $[7,8]$, or to obtain small-size but also broadband topologies [9-12]. Referring to the enhancement of the frequency bandwidth, a topology with additional transmission line sections at the coupler ports has been proposed in [7]. In this way, the bandwidth may be extended to 1.84 times as wide as the conventional ring coupler, but the printed area is increased. Other solutions to extend the bandwidth have been reported in [8-10], where topologies with coplanar waveguides and slotlines have been proposed. For these topologies, the frequency bandwidth may be extended to one octave $[8,9]$ or even 1.8 octave [10], for return-losses at the ports greater than $10 \mathrm{~dB}$. While in [8] the printed area is not reduced compared to the conventional ring coupler, in [9], the ring circumference of the coupler is reduced to $0.67 \lambda_{g}$. For the topology reported in [10] the average ring circumference is $\sim 1.1 \lambda_{g}$. Unfortunately, the coupler topologies presented in [8-10] cannot be realized with microstrip lines. A frequency bandwidth enhancement to one octave may be also obtained by replacing the $3 \lambda_{g} / 4$ transmission line section of the conventional ring coupler by a coupled-lines coupler configured as presented in [11]. Moreover, if folded stubs are used to decrease the length of the $\lambda_{g} / 4$ sections, as well as the length of the additional coupler which model the $3 \lambda_{g} / 4$ section, the circumference of the 3-dB ring coupler may be reduced to $\sim 0.7 \lambda_{g}$ and the fractional bandwidth may be increased to $\sim 30 \%$ [12], for the same performances as reported in [8-10]. Unfortunately, because of the three-dimensional structure of the additional coupler used in [12], the fabrication process is quite difficult.

A small-size ring coupler having the same frequency bandwidth as for the conventional topology, based on fully-distributed Composite Right-/Left-Handed (CRLH) phase shifters, has been investigated in detail in [13]. For this type of ring coupler, a solution for bandwidth enhancement up to one octave is proposed and analyzed in this paper. Moreover, the footprint area for the proposed ring coupler is reduced to $25 \%$ compared to the conventional ring coupler topology, for similar performances as reported in $[8-10,12]$. The proposed coupler topology may be implemented with microstrip lines using one mask technological process. Compared to other CRLH based ring couplers topologies [14, 15], the proposed topology does not include lumped elements, the footprint area is smaller and the frequency bandwidth is enhanced.

The paper is organized as follows. In Section 2, the topology for the proposed broadband and small-size coupler is introduced step by step, starting from the conventional ring coupler topology. A

\footnotetext{
Received 10 October 2013, Accepted 12 November 2013, Scheduled 19 November 2013

* Corresponding author: Stefan Simion (stefan.simion@yahoo.com).

1 Department of Electronics and Communications of Military Technical Academy, George Cosbuc 39-49, Bucharest 050141, Romania.

2 Department of Electronics Engineering of University Tor Vergata, Via del Politecnico 1, Rome 00133, Italy.
} 


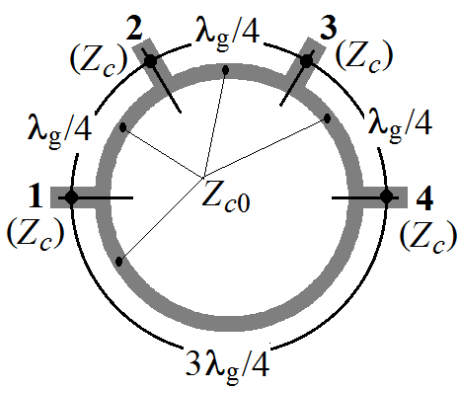

Figure 1. The conventional 3-dB ring coupler [1].

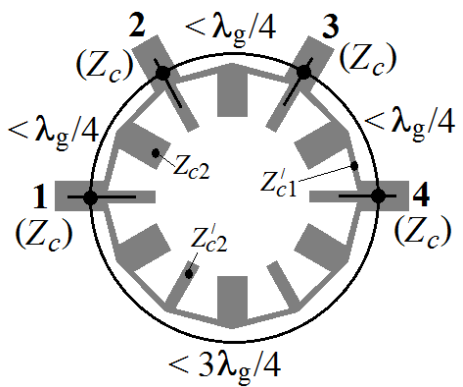

Figure 2. Compact size 3-dB ring coupler based on fully-distributed CRLH structures, analysed and designed in $[6,13]$.

design example for the proposed coupler topology is presented in Section 3, where the technological process for the fabrication of the designed coupler is presented, too. In order to validate this coupler topology, a coupler with the proposed topology is fabricated and measured. The numerical results obtained by electromagnetic analysis and the experimental results are compared in Section 4, showing that they are in good agreement.

\section{THE PROPOSED TOPOLOGY FOR THE BROADBAND AND SMALL-SIZE RING COUPLER}

The microstrip configuration of the conventional 3-dB ring coupler is presented in Fig. 1 [1], where $Z_{c 0}=\sqrt{2} Z_{c}$ and $Z_{c}$ is the characteristic impedance at the coupler ports. In order to reduce the footprint area of this device, one possibility is to replace the $\lambda_{g} / 4$ transmission line sections with CRLH based phase shifters which are shorter than $\lambda_{g} / 4[6,13]$, as it is shown in Fig. 2. The topology of these phase shifters consists of two transmission line sections of characteristic impedances $Z_{c 1}^{/}$, loaded by three stubs of characteristic impedances $2 Z_{c 2}^{/}$and $Z_{c 2}$. If $S F$ is the scaling factor defined as the ratio between the electrical length of the $90^{\circ}$ phase shifter consisting of a simple wave-quarter transmission line section, and the electrical length of the $90^{\circ} \mathrm{CRLH}$ phase shifter, the ratio between the area of the CRLH based ring coupler shown in Fig. 2 and the area of the conventional ring coupler area shown in Fig. 1 is $A R_{1}=S F^{-2}$. In Fig. 2, the electrical lengths for the transmission lines are equal to $180^{\circ} \cdot S F^{-1} / 4$, at the central working frequency. As it is demonstrated in [13], it is possible to obtain $S F>1$, therefore, a footprint area reduction is possible (i.e., $A R_{1}<1$ ). Unfortunately, as shown in [13], the frequency bandwidth for the small-size ring coupler presented in Fig. 2 is the same as for the conventional ring coupler topology presented in Fig. 1.

A solution to increase the frequency bandwidth for the small-size ring coupler presented in Fig. 2 is to replace the $270^{\circ}$ phase shifter connected between the ports 1 and 4 with an additional 3-dB coupler, as shown in Fig. 3(a). Because of the tight coupling, a Lange coupler must be used for this additional coupler. This coupler is configured as shown in Fig. 3(b), so that it works as a $270^{\circ}$ phase shifter [11]. The electrical lengths for the two shorter stubs of characteristic impedance $Z_{c 2}^{/}$from Fig. 3(a) may be computed by imposing the same input impedance for a stub of characteristic impedance $Z_{c 2}^{\prime}$ and a stub of characteristic impedance $2 Z_{c 2}^{/}$, at the central working frequency. In this way, the formula for the electrical lengths of these stubs, at the central working frequency, is $180^{\circ} / \pi \cdot \tan ^{-1}\left[0.5 \cdot \tan \left(\pi \cdot S F^{-1} / 4\right)\right]$. The ratio between the area for this proposed ring coupler topology and the area of the ring coupler shown in Fig. 2 is $A R_{2}=(0.5+S F / 6)^{2}$. An area reduction between the coupler topologies shown in Fig. 2 and Fig. 3(a) may be obtained if $A R_{2}<1$, or $S F<3$. Therefore, the ratio between the area of the ring coupler presented in Fig. 3(a) and the area of the conventional ring coupler shown in Fig. 1 is $A R=A R_{1} \cdot A R_{2}$.

In Table 1 , the values for the impedances $Z_{c 1}^{\prime}, Z_{c 2}^{\prime}\left(\right.$ for $\left.Z_{c 0}=70.7 \Omega\right)$ and $S F$ computed as presented 


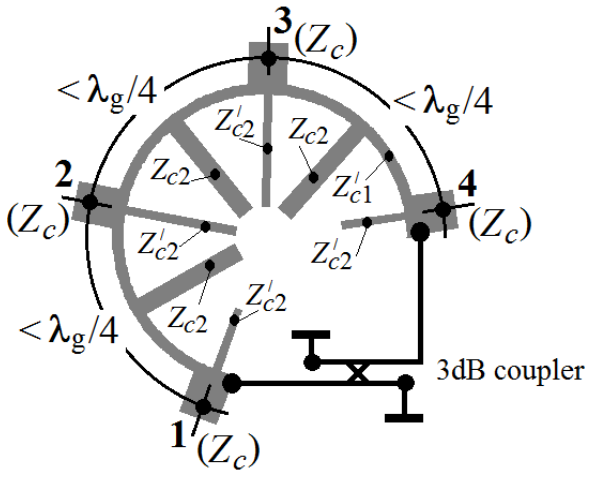

(a)

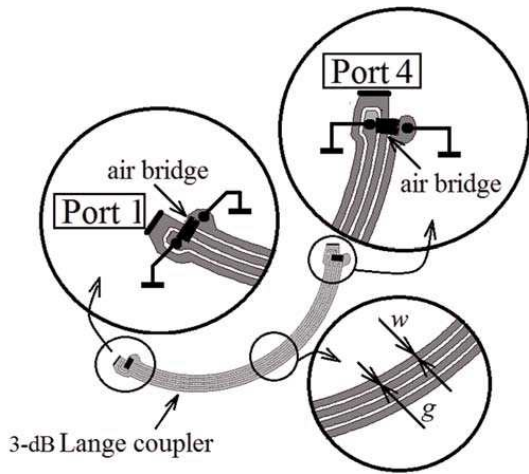

(b)

Figure 3. (a) Broadband and small-size 3-dB ring coupler, proposed in this paper and (b) the topology for the additional $3-\mathrm{dB}$ coupler configured with a Lange coupler.

in [13], but also the values for $A R_{1}, A R_{2}$ and $A R$ are given for a few values of $Z_{c 2}$. It is observed that for characteristic impedance values which may be implemented in practice, the footprint area may be reduced to $20 \%$, compared to the conventional topology may be obtained.

\section{COUPLER DESIGN AND FABRICATION}

The 3-dB ring coupler having the topology shown in Fig. 3(a) has been designed for the central working frequency of $1.5 \mathrm{GHz}$, imposing $Z_{c 2}=80 \Omega$ and $Z_{c}=50 \Omega\left(Z_{c 1}^{\prime}=102 \Omega\right.$ and $Z_{c 2}^{\prime}=115 \Omega$ - see Table 1$)$. The coupler has been designed with microstrip lines, using the substrate RT/duroid 5870 of $1.575 \mathrm{~mm}$ thickness.

The additional Lange coupler consists of four coupled lines. The width of the $50 \Omega$ transmission lines used for the ports 1 and 4 of the ring coupler must be taken into account when the length of the additional $3-\mathrm{dB}$ coupler is designed. As a result, the central frequency of the additional 3-dB coupler must be $1.7 \mathrm{GHz}$, higher than the central working frequency of the ring coupler. In this way, the phase shift introduced by the Lange coupler, including its connecting pads to the ports 1 and 4 of the ring coupler, will be equal to $270^{\circ}$, at the central working frequency of $1.5 \mathrm{GHz}$. Taking into account the substrate parameters presented above, the strips width and the gap between the strips are equal to $w=0.3 \mathrm{~mm}$ and $g=0.2 \mathrm{~mm}$ (see Fig. 3(b)). Also, the length of the Lange coupler, including the connecting pads, is equal to $L_{\text {Lange }}=31.4 \mathrm{~mm}$. The Lange coupler has been designed for the characteristic impedance at the ports equal to $70.7 \Omega$.

The layout of the CRLH based phase shifters was designed and optimized for the central working frequency, taking into account the T-junctions discontinuities. Finally, the total length of the all three CRLH based phase shifters is $L_{\mathrm{CRLH}}=78.6 \mathrm{~mm}$.

The circumference of the ring coupler is the sum of $L_{\text {Lange }}$ and $L_{\mathrm{CRLH}}$. In this way, the diameter

Table 1. Computed values for $Z_{c 1}^{\prime}, Z_{c 2}^{\prime}, S F, A R_{1}, A R_{2}$ and $A R$, for a few values of $Z_{c 2}$.

\begin{tabular}{|c|c|c|c|c|c|c|}
\hline$Z_{c 2}(\Omega)$ & $Z_{c 1}^{\prime}(\Omega)$ & $Z_{c 2}^{\prime}(\Omega)$ & $S F$ & $A R_{1}$ & $A R_{2}$ & $A R$ \\
\hline 50 & 120.7 & 85.3 & 1.77 & 0.32 & 0.63 & 0.20 \\
\hline 60 & 112.4 & 95.3 & 1.64 & 0.37 & 0.60 & 0.22 \\
\hline 70 & 106.4 & 105.3 & 1.56 & 0.41 & 0.58 & 0.24 \\
\hline 80 & 101.9 & 115.3 & 1.50 & 0.44 & 0.56 & 0.25 \\
\hline 90 & 98.5 & 125.3 & 1.44 & 0.48 & 0.55 & 0.26 \\
\hline 100 & 95.7 & 135.3 & 1.40 & 0.51 & 0.54 & 0.27 \\
\hline 110 & 93.4 & 145.3 & 1.37 & 0.54 & 0.53 & 0.28 \\
\hline
\end{tabular}




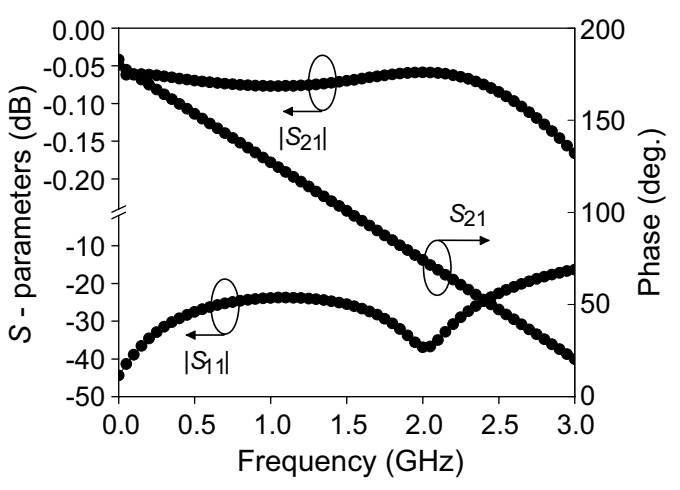

Figure 4. Numerical results for the $270^{\circ}$ phase shifter realized by using a Lange coupler configured as shown in Fig. 3(b). The results were obtained with IE3D - Zeland, for characteristic impedance at the ports equal to $70.7 \Omega$.

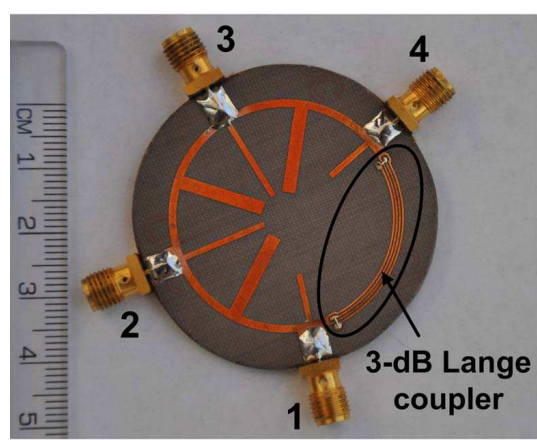

Figure 5. Photo of the fabricated broadband and small-size fully distributed CRLH based 3-dB ring coupler.

of the proposed ring coupler is equal to $35 \mathrm{~mm}$. For this diameter, the footprint area for the coupler is 0.25 of the area occupied by the conventional ring coupler topology (this value for the area reduction is the same as the expected one - see Table 1 for $Z_{c 2}=80 \Omega$, where $A R=0.25$ ).

Knowing the diameter of the ring coupler, the layout may be drawn. For the Lange coupler, the layout has been presented in Fig. 3(b), where the details around the ports are also shown. Fig. 4 shows the results obtain by analyzing the layout of the Lange coupler, by using IE3D - Zeland software. The phase shift is $+90^{\circ}$ (or equal to $270^{\circ}$ ) at $1.71 \mathrm{GHz}$. Also, the insertion loss and the return loss are less than $0.1 \mathrm{~dB}$ and greater than $25 \mathrm{~dB}$, respectively, for frequencies up to $2.4 \mathrm{GHz}$.

The fabrication process involved a standard one mask photolithographic process and wet etching to remove the copper in excess. The air-bridges for the Lange coupler, as well as the via-holes, have been realized by using wires of $0.2 \mathrm{~mm}$ diameter. For the experimental characterization, SMA connectors have been assembled at the all four coupler ports. The photo of the fabricated coupler is shown in Fig. 5.

\section{ELECTROMAGNETIC SIMULATION AND EXPERIMENTAL RESULTS}

For the electromagnetic (EM) analysis of the coupler, the software IE3D - Zeland has been used. Experimental results have been obtained using Anritsu MS2026C two-port Vector Network Analyzer (VNA). The simulated and experimental results are presented in Fig. 6. A very good agreement between them may be observed for the magnitudes of the scattering parameters, as well as for the phase difference between the output ports.

For one octave of the frequency bandwidth, from $1 \mathrm{GHz}$ to $2 \mathrm{GHz}$, the differences between the simulated and experimental results are less than $0.2 \mathrm{~dB}$ for $\left|S_{21}\right|,\left|S_{41}\right|,\left|S_{43}\right|$ and $\left|S_{23}\right|$, less than $2 \mathrm{~dB}$ for $\left|S_{31}\right|$ and $\left|S_{13}\right|$, while for $\left|S_{11}\right|$ and $\left|S_{33}\right|$ these differences are less than $4 \mathrm{~dB}$. Also, for the phase differences between $S_{21}$ and $S_{41}$ (see Phase $\left(S_{21} / S_{41}\right)$ ), as well as between $S_{43}$ and $S_{23}$ (see Phase $\left(S_{43} / S_{23}\right)$ ), the differences between the simulated and experimental results are less than $2^{\circ}$ and $0.6^{\circ}$, respectively.

Referring to the experimental results, for the frequency bandwidth from $1 \mathrm{GHz}$ to $2 \mathrm{GHz},\left|S_{21}\right|$ and $\left|S_{43}\right|$ are between $-2.9 \mathrm{~dB}$ and $-3.1 \mathrm{~dB}$, while $\left|S_{41}\right|$ and $\left|S_{23}\right|$ are between $-3.4 \mathrm{~dB}$ and $-3.7 \mathrm{~dB}$. The magnitude imbalance is less than $0.5 \mathrm{~dB}$ for the in-phase output ports, as well as for the out-of-phase output ports. Also, $\left|S_{31}\right|$ and $\left|S_{13}\right|$ are smaller than $-28 \mathrm{~dB}$, while $\left|S_{11}\right|$ and $\left|S_{33}\right|$ are smaller than $-12 \mathrm{~dB}$ and $-15 \mathrm{~dB}$, respectively. The phase difference Phase $\left(S_{21} / S_{41}\right)$ is between $178^{\circ}$ and $184^{\circ}$, while the phase difference Phase $\left(S_{43} / S_{23}\right)$ is between $-1.1^{\circ}$ and $-2.4^{\circ}$. 


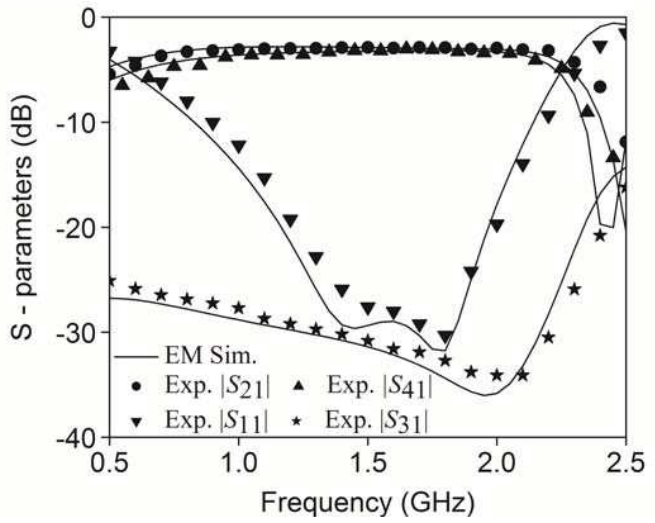

(a)

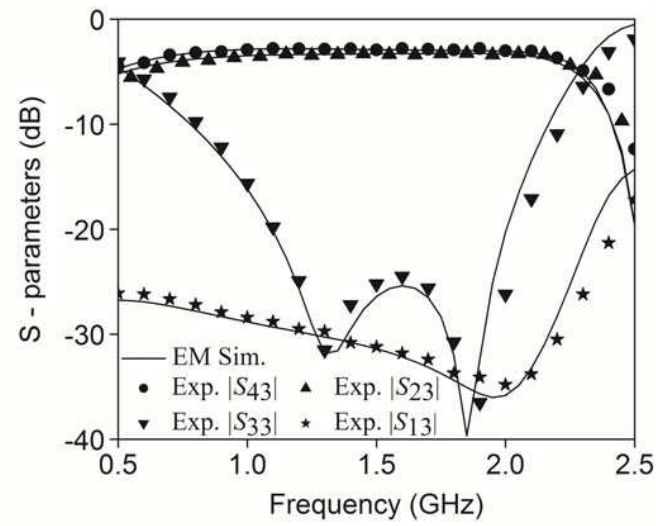

(b)

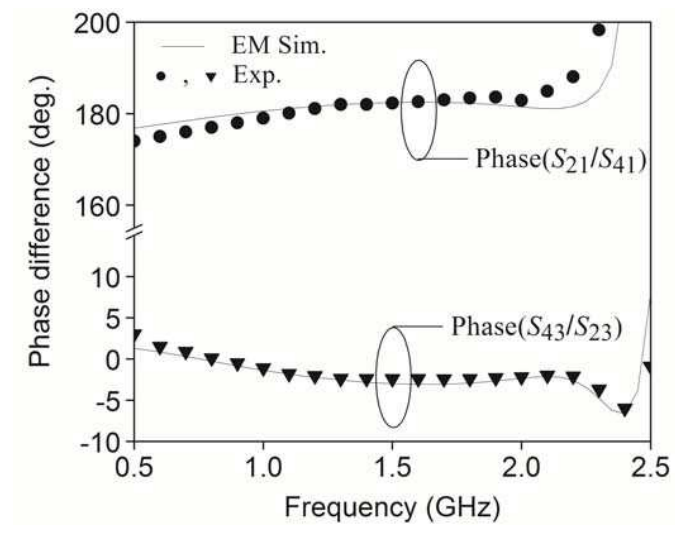

(c)

Figure 6. (a)-(b) Simulated and experimental magnitudes of the scattering parameters and (c) phase shift differences, for the designed and fabricated broadband and small-size 3-dB ring coupler.

\section{CONCLUSIONS}

A topology for a broadband and small-size 3-dB ring coupler has been proposed and validated experimentally. The coupler may be realized with microstrip lines. The fabrication involves one mask photolithographic process, and also two air-bridges and via-holes for the Lange coupler.

\section{ACKNOWLEDGMENT}

This work has been performed on a research contract having as partners the Univ. Tor Vergata from Rome and the Military Technical Academy from Bucharest, in the frame of the Executive Programme for Scientific and Technological Co-operation between the Italian Republic and Romania, for the years 2013-2014. The authors wish to acknowledge physicist Florin Craciunoiu from the Institute of Microtechnologies-Bucharest for his support concerning the circuit fabrication.

\section{REFERENCES}

1. Pon, C. Y., "Hybrid-ring directional coupler for arbitrary power divisions," IRE Trans. Microwave Theory and Techniques, Vol. 9, No. 6, 529-535, Nov. 1961.

2. Huo, J.-T. and C.-H. Tsai, "Generalized synthesis of rat race ring coupler and its application to circuit miniaturization," Progress In Electromagnetics Research, Vol. 108, 51-64, 2010. 
3. Kim, D. I. and G. S. Yang, "Design of new hybrid-ring directional coupler using $\lambda / 8$ or $\lambda / 6$ sections," IEEE Trans. on Microwave Theory and Techniques, Vol. 39, No. 10, 1179-1784, Oct. 1991.

4. Coupez, J. P., A. Peden, and C. Person, "Analysis and design of ultra miniature hybrid ring directional coupler," Proc. 22nd European Microwave Conf., Vol. 1, 443-447, Helsinki, Finland, 1992.

5. Chuang, M. L., "Miniaturized ring coupler of arbitrary reduced size," IEEE Microwave and Wireless Components Letters, Vol. 15, No. 2, 16-18, Jan. 2005.

6. Simion, S., "Small-size ring coupler design method based on fully distributed composite right/left handed approach," Electronics Letters, Vol. 48, No. 23, 1481-1483, Nov. 2012.

7. Kim, D. I. and Y. Naito, "Broad-band design of improved hybrid-ring 3-dB directional couplers," IEEE Trans. on Microwave Theory and Techniques, Vol. 30, No. 11, 2040-2046, Nov. 1982.

8. Ho, C. H., L. Fan, and K. Chang, "Broadband uniplanar hybrid ring coupler," Electronics Letters, Vol. 29, No. 1, 44-45, Jan. 1993.

9. Murgulescu, M. H., M. Moisan, P. Legaud, E. Penard, and I. Zaquine, "New wideband, $0.67 \lambda_{g}$ circumference $180^{\circ}$ hybrid ring coupler," Electronics Letters, Vol. 30, No. 4, 299-300, Feb. 1994.

10. Wang, T. and K. Wu, "Size-reduction and band-broadening design technique of uniplanar hybrid ring coupler using phase inverter for $\mathrm{M}(\mathrm{H}) \mathrm{MIC}$ 's," IEEE Trans. on Microwave Theory and Techniques, Vol. 47, No. 2, 198-206, Feb. 1999.

11. March, S., "A wideband stripline hybrid ring," IEEE Trans. on Microwave Theory and Techniques, Vol. 16, No. 6, 361, Jun. 1968.

12. Ahn, H. R. and B. Kim, "Small wideband coupled-line ring hybrids with no restriction on coupling power," IEEE Trans. on Microwave Theory and Techniques, Vol. 57, No. 7, 1806-1817, Jul. 2009.

13. Simion, S., "Design method of miniaturized ring coupler using phase shifters consisting of fullydistributed composite right/left-handed structures," Progress In Electromagnetics Research B, Vol. 50, 61-76, 2013.

14. Chi, P. L., "Miniaturized ring coupler with arbitrary power divisions based on the composite right/left-handed transmission lines," IEEE Microwave and Wireless Components Letters, Vol. 22, No. 4, 170-172, Apr. 2012.

15. Okabe, H., C. Caloz, and T. Itoh, "A compact enhanced-bandwidth hybrid ring using an artificial lumped-element left-handed transmission-line section," IEEE Trans. on Microwave Theory and Techniques, Vol. 52, No. 3, 798-804, Mar. 2004. 